\title{
Measurements and simulation of ionospheric scattering on VHF and UHF radar signals: Coherence times, coherence bandwidths, and $S_{4}$
}

\author{
Neil C. Rogers, ${ }^{1}$ Paul S. Cannon, ${ }^{1}$ and Keith M. Groves ${ }^{2}$
}

Received 10 October 2008; revised 17 July 2009; accepted 29 July 2009; published 21 October 2009.

[1] Irregularities in the electron density of the ionosphere cause phase and amplitude scintillation on transionospheric VHF and UHF radar signals, particularly at lower radio frequencies. The design of radar and other transionospheric systems requires good estimates of the coherence bandwidth (CB) and coherence time (CT) imposed by a turbulent ionosphere. $\mathrm{CB}$ and $\mathrm{CT}$ measurements of the equatorial ionosphere, made using the Advanced Research Project Agency Long-range Tracking and Identification Radar $158 \mathrm{MHz}$ and $422 \mathrm{MHz}$ phase coherent radar located on Kwajalein $\left(9.4^{\circ} \mathrm{N}, 167.5^{\circ} \mathrm{E}\right)$, are presented as a function of the two-way $\mathrm{S}_{4}$ scintillation index at $422 \mathrm{MHz}$ The $\log$ linear regression equations are $\mathrm{CT}=1.46 \exp \left(-1.40 \mathrm{~S}_{4}\right) \mathrm{s}$ at $158 \mathrm{MHz}$ and $\mathrm{CT}=2.31$ $\exp \left(-1.10 \mathrm{~S}_{4}\right) \mathrm{s}$ at $422 \mathrm{MHz}$. CT also varies by a factor of $2-3$ depending on the effective scan velocity through the ionosphere, $v_{\text {eff. }}$ The $\mathrm{CT}$ and $\mathrm{CB}$, as a function of $\mathrm{S}_{4}$, have been compared to those from the Trans-Ionospheric Radio Propagation Simulator, a phase screen model. A close agreement is achieved using appropriate values of $v_{\text {eff }}$ and midrange values of phase spectral index and outer scale. Validation of CB is, however, limited by insufficient radar chirp bandwidth. Formulating the model in terms of the two-way $\mathrm{S}_{4}$ index (an easily measurable parameter) rather than more fundamental phase screen parameters (which are difficult to obtain), improves its utility for the systems engineer. The frequency dependencies (spectral indices) of $\mathrm{S}_{4}$ and of $\mathrm{CT}$ are also presented to allow interpolation and some extrapolation of these results to other frequencies.

Citation: Rogers, N. C., P. S. Cannon, and K. M. Groves (2009), Measurements and simulation of ionospheric scattering on VHF and UHF radar signals: Coherence times, coherence bandwidths, and $\mathrm{S}_{4}$, Radio Sci., 44, RS0A33, doi:10.1029/2008RS004035.

\section{Introduction}

[2] Turbulent processes in the ionosphere create a spectrum of electron density irregularities which forward scatter VHF and UHF radio signals passing through them. The resultant phase and amplitude fluctuations (or scintillation) at the receiver limits the time and bandwidth over which the channel is coherent. The coherence time (CT) and coherence bandwidth (CB) are useful engineering parameters for the design of transionospheric radio and radar systems. For example, the ionospheric CB may limit either the data transmis-

\footnotetext{
${ }^{1}$ Centre for RF Operational Environments, QinetiQ, Malvern, UK.

${ }^{2}$ Air Force Research Laboratory, Hanscom Air Force Base, Massachusetts, USA.
}

Copyright 2009 by the American Geophysical Union. 0048-6604/09/2008RS004035 sion rate in a satellite communications link or the range resolution of a transionospheric radar. The ionospheric CT may limit the number of pulses available for coherent pulse integration, which could, for example, limit the along-track resolution in a space-based synthetic aperture radar image [van de Kamp et al., 2009; Belcher and Rogers, 2009].

[3] Studies of scintillation over a two-way (radar) path are relatively uncommon due to the expensive radar facilities required; but see, for example, papers by Knepp [1983a, 1983b] and Coster et al. [1993, 2002] who have addressed the impact of the ionosphere on radar phasecoherent pulse integration. This paper extends the earlier work by Cannon et al. [2006] and a companion paper [Rogers et al., 2009]. It uses the measurements from the Advanced Research Project Agency (ARPA) Long-range Tracking and Identification Radar (ALTAIR) radar in association with the Trans-Ionospheric Radio Propagation Simulator (TIRPS) [Rogers et al., 2009] to under- 
Table 1. Dates, Times, Maximum Elevations, and Number of CSFs for Passes of Satellite 2826

\begin{tabular}{ccccc}
\hline Date & UT Range & Local Time Range & Maximum Elevation (deg) & Number of CSFs (Above 20 Elevation) \\
\hline 18 Jan 2005 & $1003-1015$ & $2113-2125$ & 49.5 & 115 \\
21 Oct 2005 & $1027-1042$ & $2137-2152$ & 81.1 & 128 \\
25 Oct 2005 & $0941-0956$ & $2051-2106$ & 86.5 & 130 \\
14 Sep 2006 & $1115-1121$ & $2225-2231$ & 48.4 & 108 \\
15 Sep 2006 & $1036-1044$ & $2146-2154$ & 85.9 & 128 \\
& & & & Total $=609$ \\
\hline
\end{tabular}

stand the relationships between $\mathrm{CT}$ and $\mathrm{CB}$ and parameters such as $\mathrm{S}_{4}$. In so doing we have been able to further refine the TIRPS model and we gain insight into a number of important issues.

\section{Wideband Ionospheric Distortion Experiment}

[4] The measurements in this study were collected as part of the joint UK-U.S. Wideband Ionospheric Distortion Experiment (WIDE) [Cannon et al., 2006]. WIDE uses the ALTAIR monostatic radar located on Kwajalein $\left(9.4^{\circ} \mathrm{N}, 167.5^{\circ} \mathrm{E}\right)$ to track orbiting calibration spheres. Linear FM chirps were transmitted coherently at $158 \mathrm{MHz}$ (7 MHz bandwidth) and $422 \mathrm{MHz}(18 \mathrm{MHz}$ bandwidth) with a pulse repetition frequency (PRF) in the range $285 \pm$ $50 \mathrm{~Hz}$ and with PRF changes occurring no more than once every $20 \mathrm{~s}$. The complex received signal was recorded for analysis as described below. For these studies we used only object 2826 , which is at an orbital altitude of $767 \pm 6 \mathrm{~km}$ altitude and at $70^{\circ}$ inclination.

[5] After proof-of-concept trials in January 2005, subsequent measurements took place during the AugustOctober 2005 and 2006 seasonal peak in scintillation occurrence at Kwajalein [Aarons, 1993]. Late evening passes, when the likelihood of strong scintillation was greatest [e.g., Aarons, 1982], and passes with high maximum elevation were selected. Although the measurements were taken near the minimum of the solar activity cycle, strong scintillation was nonetheless observed on several occasions, so providing a wide range of conditions. For each pass the data were processed to generate a series of scattering functions (CSFs) [Cannon et al., 2006; Rogers et al., 2009] from which the CB and CT could be derived.

[6] Each CSF was calculated from sequences of 1024 contiguous radar chirp returns which, at an average pulse repetition frequency (PRF) of $285 \mathrm{~Hz}$, constitute a $3.6 \mathrm{~s}$ sample. The component of pulse spreading due to ionospheric dispersion was removed by calculating the total electron content (TEC) from the differential group delay of the 158 and $422 \mathrm{MHz}$ chirp returns. Measurements presented in this paper were derived from channel scattering functions (CSFs) for five passes of object 2826, giving a total of 609 CSFs (see Table 1). Note that the local time at Kwajalein is $11 \mathrm{~h} 10 \mathrm{~m}$ ahead of UT. Data for elevations below $20^{\circ}$ were excluded from the measurements to minimize the impact of ground multipath and any tropospheric perturbations. This also ensures that the radar tracker had established a good lock on the target. Signalto-noise ratios ranged between 9 and $53 \mathrm{~dB}$.

[7] Under a homogeneous ionosphere (with no irregularities), the CSF profile would be confined to a single Doppler bin at $0 \mathrm{~Hz}$ (the Doppler bin width is $1 / 3.6 \mathrm{~s}=$ $0.28 \mathrm{~Hz}$ ). The delay profile would exhibit a sinc-squared profile (the linear chirp autocorrelation function) convolved with the Hann filter. This results in a $3 \mathrm{~dB}$ width $\approx 1.5 / 2 B$, where $B$ is the chirp bandwidth(=107 ns at $158 \mathrm{MHz}$ and $42 \mathrm{~ns}$ at $422 \mathrm{MHz}$ ).

\section{Trans-Ionospheric Radio Propagation Simulator}

[8] The Trans-Ionospheric Radio Propagation Simulator (TIRPS) has been developed to simulate WIDE measurements. The model, based on a thin phase screen, parabolic equation propagation technique [Knepp, 1983a, 1983b], is described in detail by Rogers et al. [2009]. The phase screen, which represents the effect of ionospheric irregularities, is defined to be a log-log linear spectrum (equation (1)) which flattens off for irregularities greater than an outer scale size $L_{o}$.

$$
\begin{aligned}
S_{\phi}(\kappa)= & {\left[\frac{1}{4} \pi^{-\frac{3}{2}} r_{e}^{2} \lambda^{2} G \cdot C_{k} L \sec (\theta)\left(\frac{2 \pi}{1000}\right)^{p+1} \frac{\Gamma\left(\frac{p}{2}\right)}{\Gamma\left(\frac{p+1}{2}\right)}\right] } \\
& \cdot \frac{1}{\left(\kappa_{0}^{2}+\kappa^{2}\right)^{p / 2}}
\end{aligned}
$$

where $\kappa=$ spatial frequency, $r_{e}=$ classical electron radius, $\lambda=$ radio wavelength, $G$ is a geometric enhancement factor [see Rino, 1979], $C_{k} L=$ vertical integrated strength of turbulence at the $1 \mathrm{~km}$ scale, $\theta=$ propagation zenith angle at the ionospheric pierce point (IPP), $p=$ phase spectral index, $\Gamma=$ Euler's gamma function and $\kappa_{0}=$ outer scale frequency $=2 \pi / L_{o}$. Equation (1) is derived from equation (16) of Rino [1979] where we have changed the dependent variable from temporal to spatial frequency and 


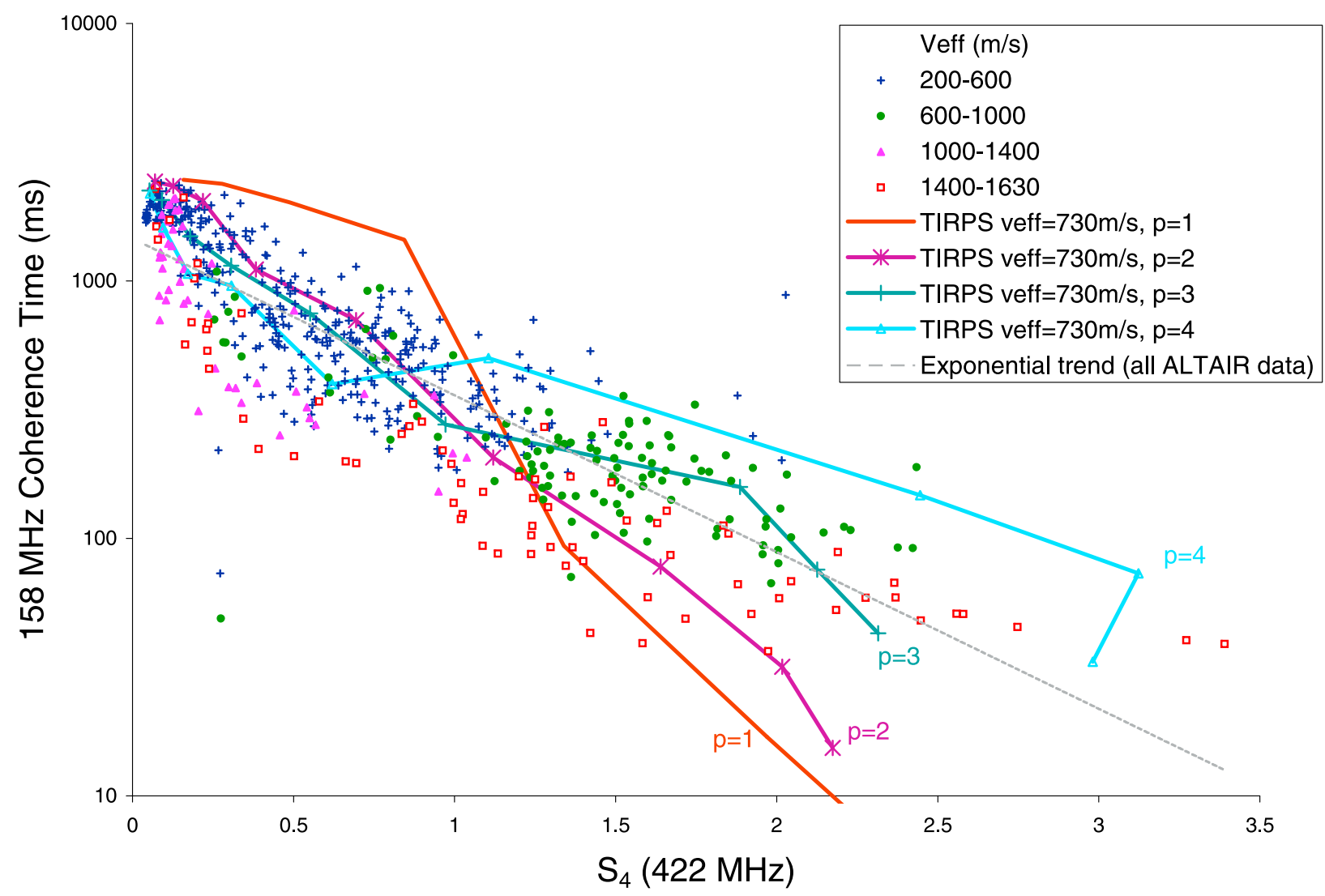

Figure 1. CT measurements for $158 \mathrm{MHz}$ CSFs as a function of the $422 \mathrm{MHz} \mathrm{S}_{4}$ index. Points represent measured CSF and are categorized by ranges of $v_{\text {eff. }}$ Mean simulated values are shown for varying spectral index $p$ (solid lines).

used the definition $C_{k} L=C_{s} L(1000 / 2 \pi)^{\mathrm{p}+1}$. In the simulations the three important variables are $p, L_{o}$ and $\left(G . C_{k} L \cdot \sec (\theta)\right)$, the latter combination having been treated as a single variable parameter.

[9] TIRPS uses Rino's equation to define the phase screen spectrum only. This uses the assumption that a thin phase screen adequately represents the extended scattering region, as demonstrated by Bramley [1977]. Thereafter the Parabolic Equation (PE) approximation [Levy, 2000] is used to describe the wave propagated through free space up to orbit height. This approximation to the full electromagnetic wave equation assumes propagation close to the line-of-sight axis, and assumes that refractive index fluctuations $\Delta \mathrm{n} / \mathrm{n} \ll 1$ (which implies fractional electron density variations $\left.\Delta \mathrm{N}_{\mathrm{e}} / \mathrm{N}_{\mathrm{e}} \ll 1\right)$. Errors in the standard $\mathrm{PE}$ approximation due to off-axis propagation are of the order $10^{-3}$ at $10^{\circ}$ off-axis [Levy, 2000, p. 11] and so are negligible for the ALTAIR radar geometry which has a $3 \mathrm{~dB}$ beam width of approximately $3^{\circ}$ at $158 \mathrm{MHz}$ or $1^{\circ}$ at $422 \mathrm{MHz}$. Assuming a typical postsunset $F$ region electron density of $10^{11} \mathrm{~m}^{-3}$, the root-mean-squared
$\Delta \mathrm{N}_{\mathrm{e}} / \mathrm{N}_{\mathrm{e}}$ is less than 0.2 for $C_{k} L<=10^{33}$ rising to 0.6 at $C_{k} L=10^{34}$ (based on Rino's definition of $C_{s}$ (equation (7) of Rino [1979]) and assuming a scattering path length $L=200 \mathrm{~km}, L_{o}=10 \mathrm{~km}$, and $\left.\mathrm{p}=2.5\right)$. The assumption $\Delta \mathrm{N}_{\mathrm{e}} / \mathrm{N}_{\mathrm{e}} \ll 1$ in the PE method may therefore become unrealistic above around $C_{k} L=10^{33}$. In this paper we have not attempted to estimate the scale of the associated errors as this would require the implementation of more complex numerical techniques for solving the full electromagnetic wave equation. Nonetheless the TIRPS model has been shown by Rogers et al. [2009] to provide reasonable approximations to the observed channel scattering functions even at high $C_{k} L$ values.

\section{Coherence Time and Coherence Bandwidth and $\mathrm{S}_{4}$ Measurement and Simulation}

[10] Following Cannon et al. [2006] and Cannon and Bradley [2003], we have chosen to define the 
Table 2. Regression Equations for Various Ranges of $v_{\text {eff }}$ for $\mathrm{CT}$ at 158 and $422 \mathrm{MHz}^{\mathrm{a}}$

\begin{tabular}{cccc}
\hline $\begin{array}{c}\text { Frequency } \\
(\mathrm{MHz})\end{array}$ & $\begin{array}{c}v_{\text {eff }} \text { Range } \\
\left(\mathrm{m} \mathrm{s}^{-1}\right)\end{array}$ & $\begin{array}{c}\text { Regression Equation } \\
\text { for CT (s) }\end{array}$ & $\begin{array}{c}\text { CT Regression Value } \\
\text { at } \mathrm{S}_{4}=1.0(\mathrm{~s})\end{array}$ \\
\hline 158 & $200-600$ & $1.70 \exp \left(-1.46 \mathrm{~S}_{4}\right)$ & 0.39 \\
158 & $1400-1630$ & $0.74 \exp \left(-1.19 \mathrm{~S}_{4}\right)$ & 0.23 \\
422 & $200-600$ & $2.19 \exp \left(-0.83 \mathrm{~S}_{4}\right)$ & 0.95 \\
422 & $1400-1630$ & $1.55 \exp \left(-1.17 \mathrm{~S}_{4}\right)$ & 0.48 \\
\hline
\end{tabular}

${ }^{\mathrm{a}} \mathrm{S}_{4}$ is the two-way $\mathrm{S}_{4}$ at $422 \mathrm{MHz}$. The regression equations are evaluated for $\mathrm{S}_{4}=1$.

coherency time (CT) and coherency bandwidth (CB) as follows:

$$
\begin{gathered}
C T=\frac{1}{\Delta B} \\
C B=\frac{1}{2 \pi \Delta T}
\end{gathered}
$$

where $\Delta \mathrm{B}$ is the Doppler spread and $\Delta \mathrm{T}$ is the delay spread, both of which are determined from the CSFs. Doppler spreads are calculated for the central $68.3 \%$ of the total power in the CSF (integrated across all delay samples) as this represents the root-mean-squared spread for a Normal power distribution, equivalent to $-2.2 \mathrm{~dB}$ from the peak. The spread is calculated as the difference between the $50+(68.3 / 2)$ percentiles of two cumulative distributions, one summed in the positive delay direction and the other in the negative delay direction. To avoid integrating noise in the measured CSFs, only those points within $6 \mathrm{~dB}$ of the peak were used in the power summation. Delay spreads were calculated in a similar manner. The $\mathrm{S}_{4}$ index (the ratio of the standard deviation of signal intensity to its mean) was calculated over $10 \mathrm{~s}$ periods (i.e., the central time of the CSF $\pm 5 \mathrm{~s}$ ). This period is somewhat shorter than is conventional for GPS satellite measurements and represents a compromise between averaging over many Fresnel zones and minimizing the spatial blurring which is inevitable for a fast moving LEO satellite.

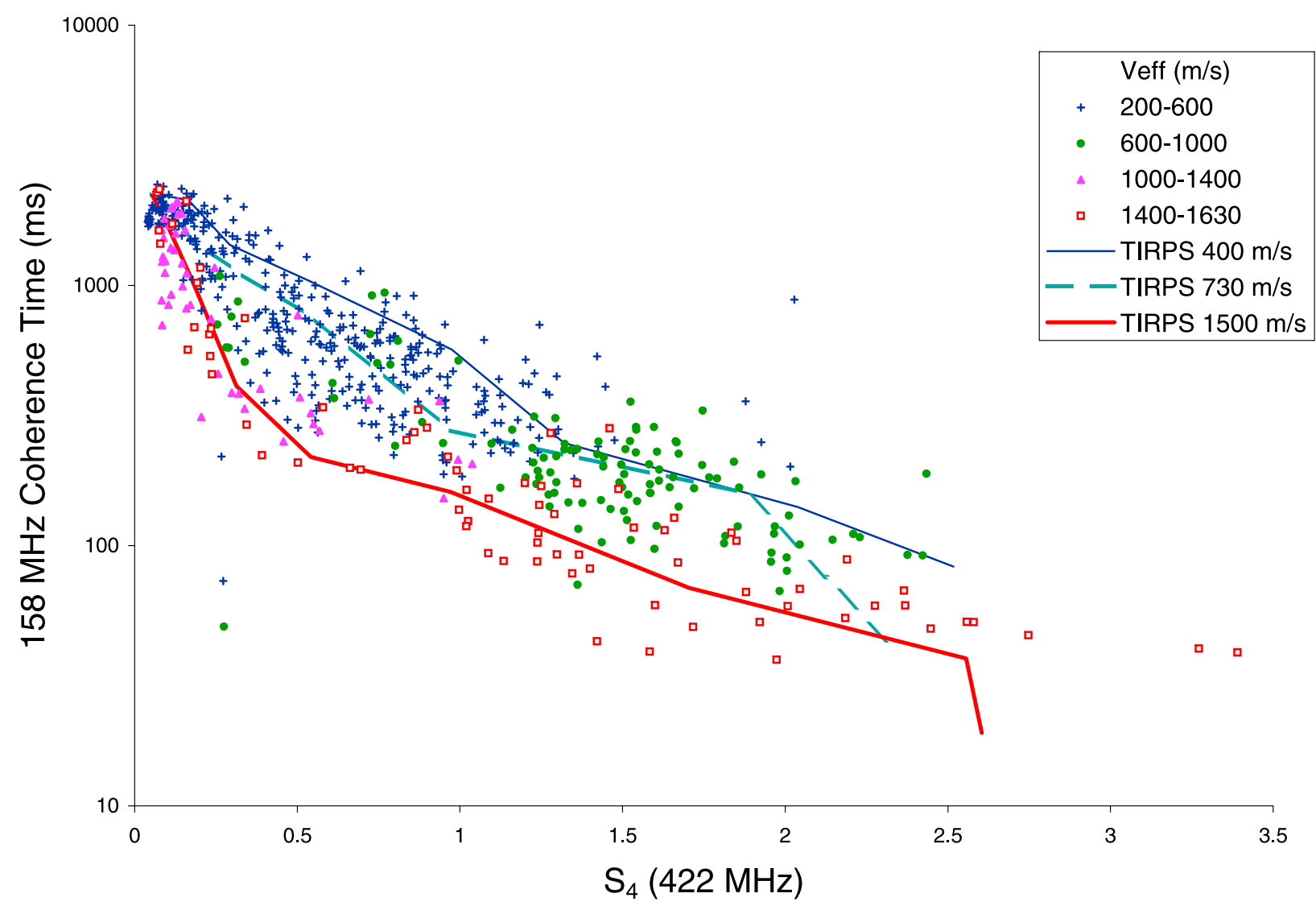

Figure 2. CT measurements for $158 \mathrm{MHz}$ CSFs as a function of the $422 \mathrm{MHz} \mathrm{S}_{4}$ index. Points represent measured CSF and are categorized by ranges of $v_{\text {eff. }}$ Mean simulated values are shown for three values of $v_{\text {eff. }}$ The phase screen spectrum (equation (1)) used $p=3$ and $L_{o}=10 \mathrm{~km}$. 


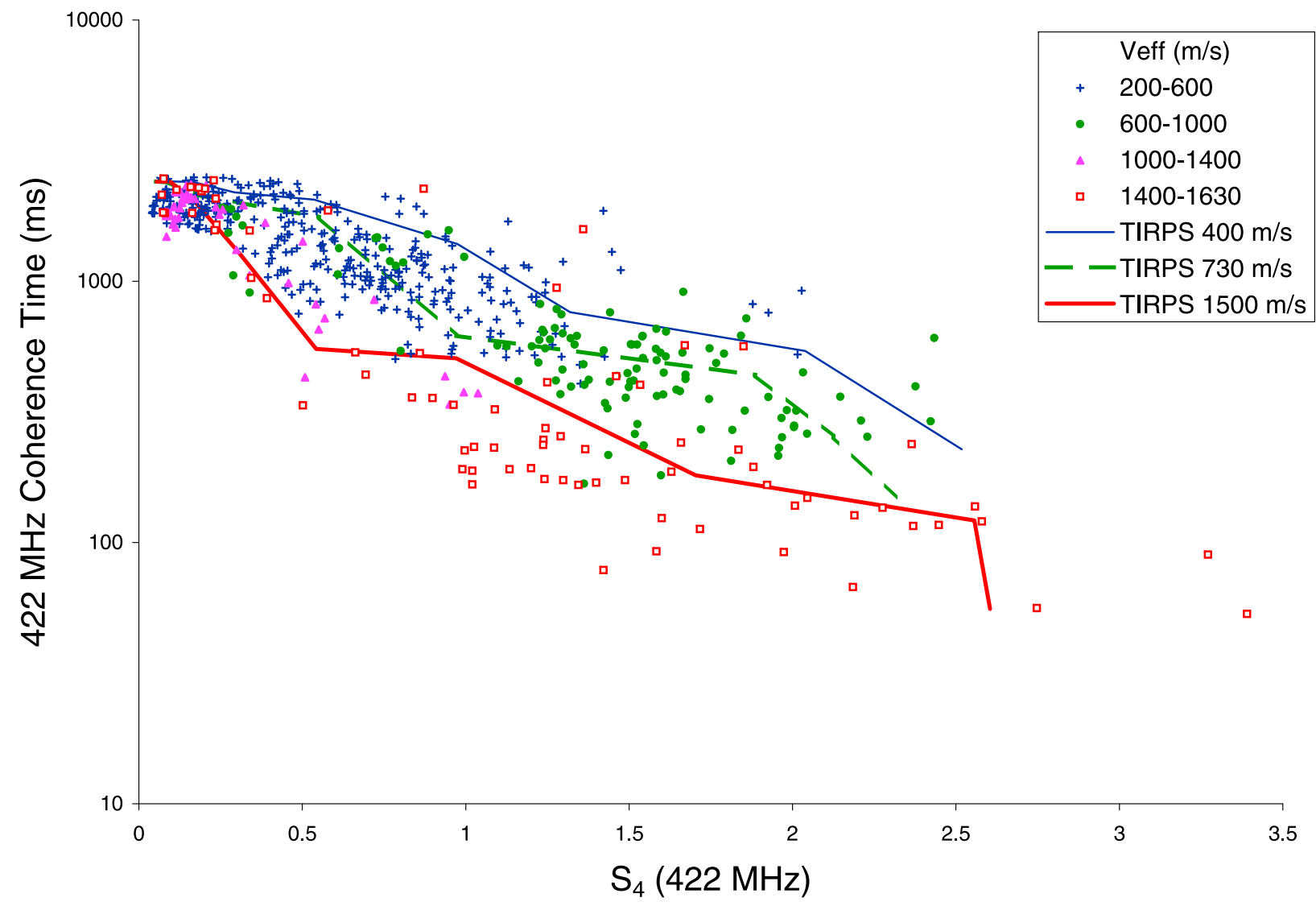

Figure 3. CT measurements for $422 \mathrm{MHz}$ CSFs as a function of the $422 \mathrm{MHz} \mathrm{S}_{4}$ index. Points represent measured CSF and are categorized by ranges of $v_{\text {eff. }}$ Mean simulated values are shown for three values of $v_{\text {eff. }}$ The phase screen spectrum (equation (1)) used $p=3$ and $L_{o}=10 \mathrm{~km}$.

[11] Figure 1 presents a log linear scatterplot of CT for $158 \mathrm{MHz}$ CSFs against the $422 \mathrm{MHz} \mathrm{S}_{4}$ index. Taking all measurements together, there is a close to exponential decline in $\mathrm{CT}$ with $\mathrm{S}_{4}$. The log linear regressions (gray dashed line in Figure 1 for $158 \mathrm{MHz}$ ) are represented by the equations:

$$
\begin{aligned}
& \mathrm{CT}_{(158 \mathrm{MHz})}=1.46 \exp \left(-1.40 \mathrm{~S}_{4(422 \mathrm{MHz})}\right)(\mathrm{s}) \\
& \mathrm{CT}_{(422 \mathrm{MHz})}=2.31 \exp \left(-1.10 \mathrm{~S}_{4}(422 \mathrm{MHz})\right)(\mathrm{s})
\end{aligned}
$$

Each point in Figure 1 has also been categorized into one of four ranges of the effective scan velocity, $v_{\text {eff }}$ [Rino, 1979]. (Note that $v_{\text {eff }}$ was generally much lower than the ionospheric penetration point (IPP) horizontal scan speed since each pass was aligned close to the magnetic meridian (i.e., along the irregularities).) It is clear that the data are well ordered by $v_{\text {eff }}$ with a very useful reduction in spread. The corresponding log linear regression equations are shown in Table 2 for two $v_{\text {eff }}$ ranges in the two frequency bands. Table 2 indicates, very broadly, that at both frequencies, a threefold to fourfold increase in $v_{\text {eff }}$ results in a twofold increase in CT at $\mathrm{S}_{4}=1.0$.

[12] The solid lines in Figure 1 represent mean values obtained from 10 random realizations of the TIRPS model, each line representing a different fixed value of spectral index, $p$. Each point on the line is connected in order of increasing $\log _{10}\left(G . C_{k} L \sec (\theta)\right)$ from 32 to 36 in increments of 0.5 , resulting in generally increasing $\mathrm{S}_{4}$. A $v_{\text {eff }}$ of $730 \mathrm{~m} \mathrm{~s}^{-1}$ (the mean of all $v_{\text {eff }}$ values), an outer scale of $10 \mathrm{~km}$ and a midrange elevation of $30^{\circ}$ are assumed. Choosing $p=3$ provides a good match to the composite exponential fit, with a similarly good fit observed in the $422 \mathrm{MHz}$ data (dashed line in Figure 3).

[13] Figure 2 reproduces the same ALTAIR measurements of CT but the superposed lines describe TIRPS simulations with fixed $p=3$ and varying $v_{\text {eff. }}$. As previously described, measurements with greater $v_{\text {eff }}$ have lower CT values and this trend is reproduced in the 


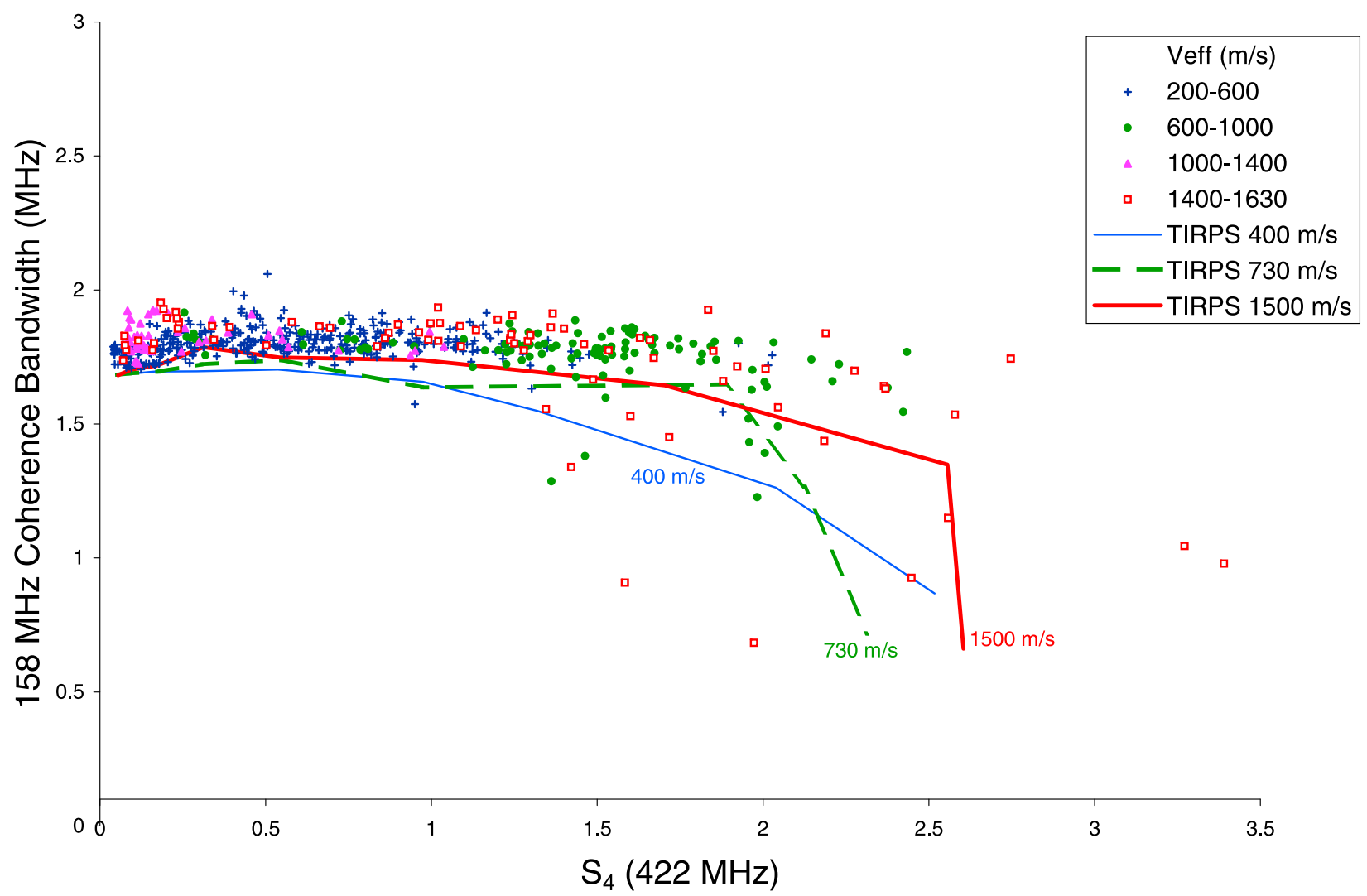

Figure 4. $\mathrm{CB}$ measurements for $158 \mathrm{MHz} \mathrm{CSFs}$ as a function of the $422 \mathrm{MHz} \mathrm{\textrm {S } _ { 4 }}$ index. Points represent measured CSF and are categorized by ranges of $v_{\text {eff. }}$. Mean simulated values are shown for three values of $v_{\text {eff. }}$. The phase screen spectrum (equation (1)) used $p=3$ and $L_{o}=10 \mathrm{~km}$.

simulations. For example, the lower red line $\left(v_{\text {eff }}=\right.$ $1500 \mathrm{~m} \mathrm{~s}^{-1}$ ) in Figure 2 provides a good fit to measurements in the category $v_{\text {eff }}=1400-1630 \mathrm{~m} \mathrm{~s}^{-1}$ (red squares) and similarly the other simulated TIRPS predictions pass through the appropriate range of ALTAIR measurements (though perhaps less well for the lowest $v_{\text {eff }}$ range). It is apparent that the inclusion of $v_{\text {eff }}$ is a necessary prerequisite of any modeling procedure of the coherency time; without it, CT predictions may be in error by factors of between 2 and 3 .

[14] Similar good agreements are observed between the $422 \mathrm{MHz}$ simulations and measurements (Figure 3), although the $422 \mathrm{MHz}$ coherence times were upper limited by the CSF measurement period ( $\sim 3.6 \mathrm{~s})$ for $\mathrm{S}_{4}<0.5$. (A similar limitation is evident in the $158 \mathrm{MHz}$ graphs but only for the lowest $\mathrm{S}_{4}<\sim 0.2$.)

[15] Coherence bandwidths (CB) are presented in Figure 4 for the $158 \mathrm{MHz}$ measurements (points) and for the TIRPS simulations parameterized as in Figure 2. These measurements are upper limited due to the insufficient bandwidth of the radar waveform, and only in the $158 \mathrm{MHz}$ data and above $\mathrm{S}_{4}=1.3$ did the $\mathrm{CB}$ drop significantly below this system-imposed limit. The TIRPS model (solid lines in Figure 4) reproduces this behavior reasonably well, at least for higher values of the $v_{\text {eff }}$ parameter. The CB for the $422 \mathrm{MHz}$ signals (not shown) fell below the system bandwidth on only three occasions.

[16] It should be noted from observations and simulations of the CSF presented by Rogers et al. [2009] that the delay spread of the waveform under strong turbulence conditions is most evident at intensities below the $-6 \mathrm{~dB}$ noise threshold used in this analysis. While these components would contribute little to the overall coherence bandwidth, in a radar system their presence may raise the clutter background, reducing the probability of detecting smaller secondary targets in the vicinity of the primary target.

\section{Radio Frequency Dependencies of $S_{4}$ and Coherence Time}

\section{1. $S_{4}$ Index}

[17] The results above, relate CT (and to some extent $\mathrm{CB})$ to $\mathrm{S}_{4}$ at just two spot frequencies. To generalize the 


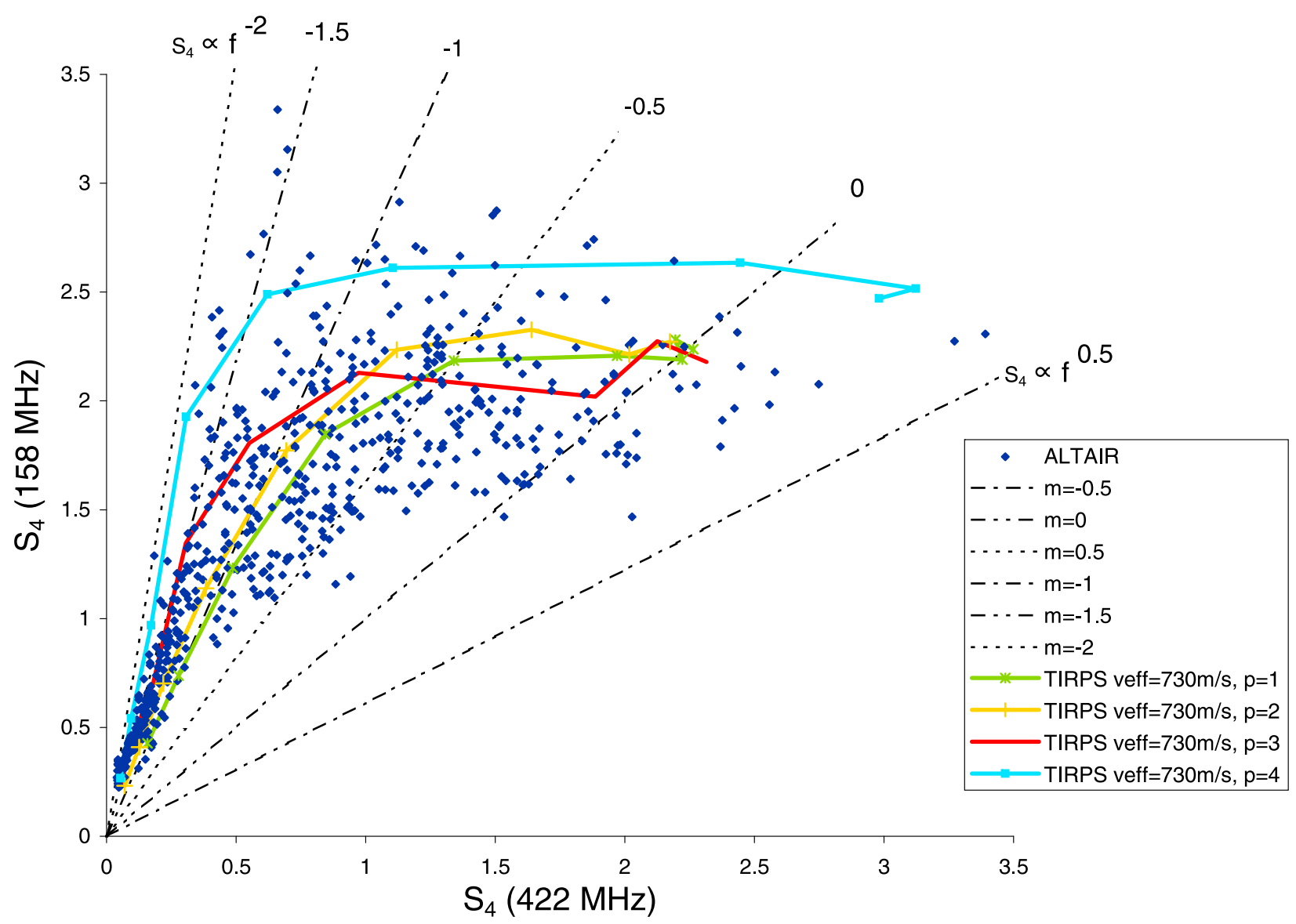

Figure 5. $\mathrm{S}_{4}(158 \mathrm{MHz})$ versus $\mathrm{S}_{4}(422 \mathrm{MHz})$ as measured from the ALTAIR radar (points) for 10 -s periods coinciding with CSF measurements. Superposed solid lines represent the mean ( \pm 1 standard deviation) of 10 TIRPS simulations at $\log _{10}\left(G . C_{k} L \cdot \sec (\theta)\right.$ ) values ranging from 32 to 36 and for four values of the spectral index $p$. Dashed diagonal lines indicate spectral indices of $\mathrm{S}_{4}$.

results to other frequencies it is necessary to examine the variation of $\mathrm{CT}$ and $\mathrm{S}_{4}$ with frequency. Published observations show that, in the case of low scintillation $\left(\mathrm{S}_{4 \text { (one-way) }}<0.5\right)$ the $\mathrm{S}_{4}$ index follows an approximately $\log$-log linear relation to the radio frequency $f$, i.e., $\mathrm{S}_{4} \propto f^{-m_{S_{4}}}$, where $m_{S 4}$ denotes the spectral index for $\mathrm{S}_{4}$ which varies between 1.6 for low $\mathrm{S}_{4}$ and 0.8 for high $\mathrm{S}_{4}$ on one-way paths [Davies, 1990, p. 290, and references therein].

[18] In Figure 5 the $\mathrm{S}_{4}$ values for $158 \mathrm{MHz}$ signals are plotted against $\mathrm{S}_{4}$ on the simultaneously recorded $422 \mathrm{MHz}$ signals. Diagonals on this plot indicate the value of $-m_{S 4}$. The same data have been replotted in Figure 6 which presents $m_{S 4}$ as a function of the twoway $422 \mathrm{MHz} S_{4}$ index. At low $\mathrm{S}_{4}$ the points follow the diagonal for $m_{S 4}=1.5 \pm 0.5$, while at higher $\mathrm{S}_{4}$ the spectral index reduces. A linear regression to the measurements (dashed gray line) in Figure 6 suggests a two-way
$\mathrm{S}_{4}$ spectral index of $m_{S_{4}}=1.53-0.80 \mathrm{~S}_{4}(2$-way, $422 \mathrm{MHz})$ although the points are widely distributed about this line and the linear fit is poor above $\mathrm{S}_{4}$ (2-way, $\left.422 \mathrm{MHz}\right)=2$ where the $422 \mathrm{MHz}$ waveform exhibits greater $\mathrm{S}_{4}$ than the $158 \mathrm{MHz}$ waveform (i.e., $\mathrm{m}_{\mathrm{S} 4}<0$ ).

[19] The TIRPS model was also used to determine the spectral index of $\mathrm{S}_{4}$ as a function of $\mathrm{S}_{4}$ at the band center frequency. The solid line in Figure 6 represents these TIRPS simulated values (mean \pm one standard deviation of 10 simulations) at each value of $C_{k} L$ with $p=3$ and $L_{o}=$ $10 \mathrm{~km}$ and $v_{\text {eff }}=730 \mathrm{~m} \mathrm{~s}^{-1}$. The simulated $\mathrm{m}_{\mathrm{S} 4}$ closely follow the linear trend observed in the measurements, reducing from approximately 1.5 at $\mathrm{S}_{4}=0$ to zero at $\mathrm{S}_{4}=$ 2.0. The slope of this line is slightly less negative above $\mathrm{S}_{4}=2$, as is also observed in the measurements.

[20] The solid lines in Figure 5 describe the TIRPS $\mathrm{S}_{4}$ values, where each line represents a different value of $p$. The simulated $\mathrm{S}_{4}$ index increased with increasing $\mathrm{C}_{\mathrm{k}} \mathrm{L}$ 


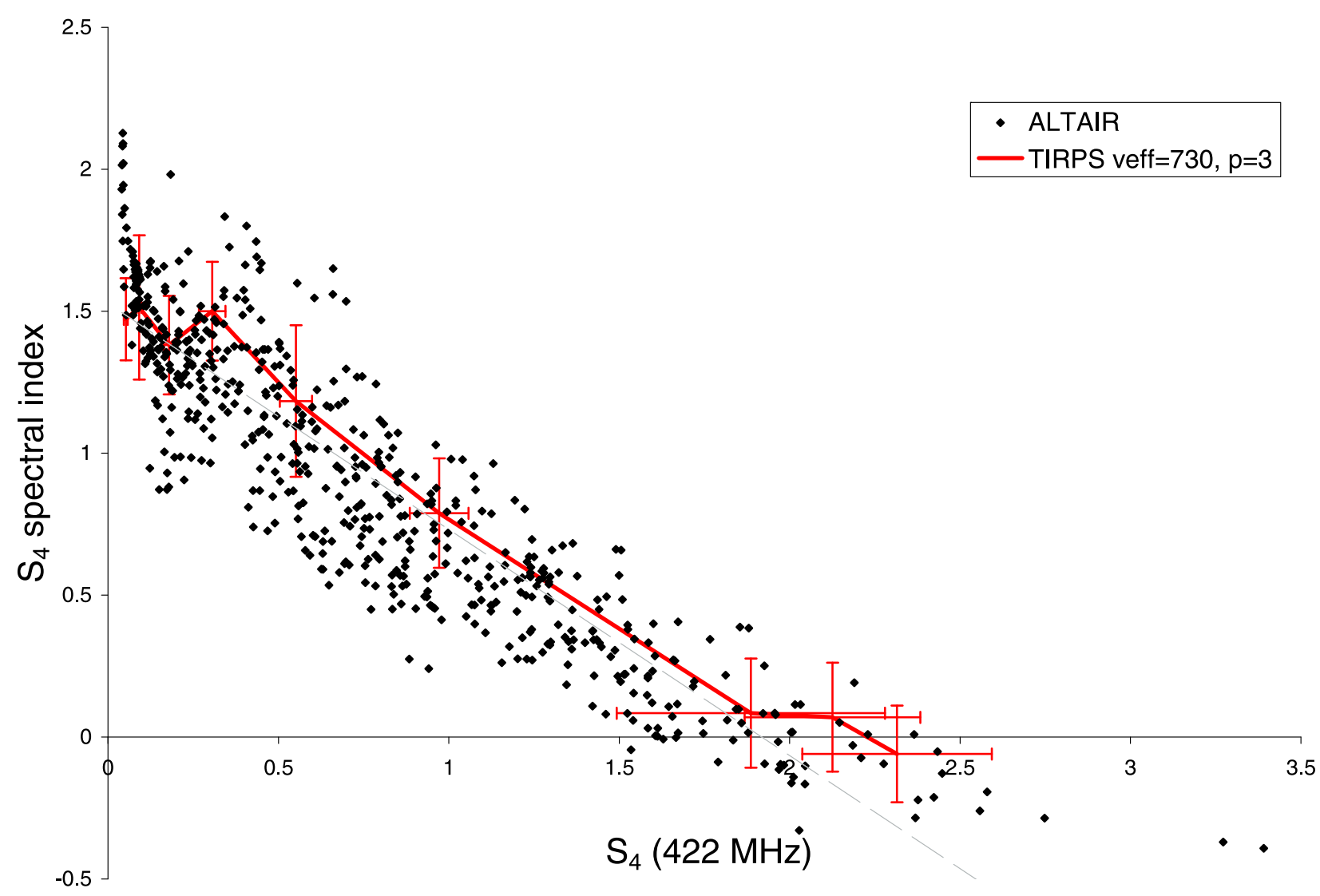

Figure 6. Spectral index of $\mathrm{S}_{4}$ as a function of the $422 \mathrm{MHz} \mathrm{\textrm {S } _ { 4 }}$ index. Dots represent calculations from ALTAIR measurements of the 2826 satellite. The solid line represents the mean $( \pm 1$ standard deviation) of 10 TIRPS simulations at $\log _{10}\left(G \cdot C_{k} L \cdot \sec (\theta)\right)$ values ranging from 32 to 36 , with $p=3$, $v_{\text {eff }}=730 \mathrm{~m} \mathrm{~s}^{-1}$, and $L_{o}=10 \mathrm{~km}$. The gray dashed line shows the linear regression to the ALTAIR measurements.

but saturated close to 2.2 for a spectral index $p<=3$, while higher values of $\mathrm{S}_{4}$ are realizable for $p>3$. Fremouw and Ishimaru [1992] showed that a limit of $\mathrm{S}_{4}=2.2(=\sqrt{ } 5)$ would be expected for a Rayleigh fading signal on perfectly correlated up and down paths. $\mathrm{S}_{4}$ greater than 2.2 is possible under conditions of strong focusing which can only be reproduced in highly anisotropic media using spectra with $p>3$ [Rino, 1980]. The theoretical maximum $\mathrm{S}_{4}$ is $3.3(=\sqrt{11})$ for Nakagami- $m$ fading [Nakagami, 1960] on perfectly correlated up and down paths [Fremouw and Ishimaru, 1992] and in the ALTAIR data set only one point exceeded this value (maximum $\left.\mathrm{S}_{4}(422 \mathrm{MHz})=3.4\right)$.

\subsection{Coherence Time}

[21] Coherence times for the $158 \mathrm{MHz}$ signals are plotted against those recorded simultaneously at $422 \mathrm{MHz}$ in Figure 7. Diagonals in Figure 7 indicate the spectral index assuming a power law relationship with frequency.
The same data are presented in Figure 8 which shows the spectral index of CT as a function of the $\mathrm{S}_{4}$ index at $422 \mathrm{MHz}$. Below $\mathrm{S}_{4} \sim 0.5$ the results are artificially influenced by the finite sample duration (as discussed in section 4) and may be ignored. At higher $\mathrm{S}_{4}$ there is a wide distribution in the spectral index from 0 to -2 , centered about $\sim-0.9$ (indicated by the 30 -point moving average (gray line in Figure 8)). The spread of the distribution narrows toward higher $\mathrm{S}_{4}$ (see Figure 8) and lower CT (see Figure 7). These results compare well with a coherence time spectral index of -1 which was predicted from the analytical model of Knepp [1983b], who assumed a Gaussian mutual coherence function and a $\kappa^{-4}$ spectrum of three-dimensional electron density (equivalent to a phase spectral index, $p$ of 3 ).

[22] The red solid lines in Figures 7 and 8 represent the ensemble average predictions from the TIRPS model using a spectral index $p=3$ and a mean effective velocity 


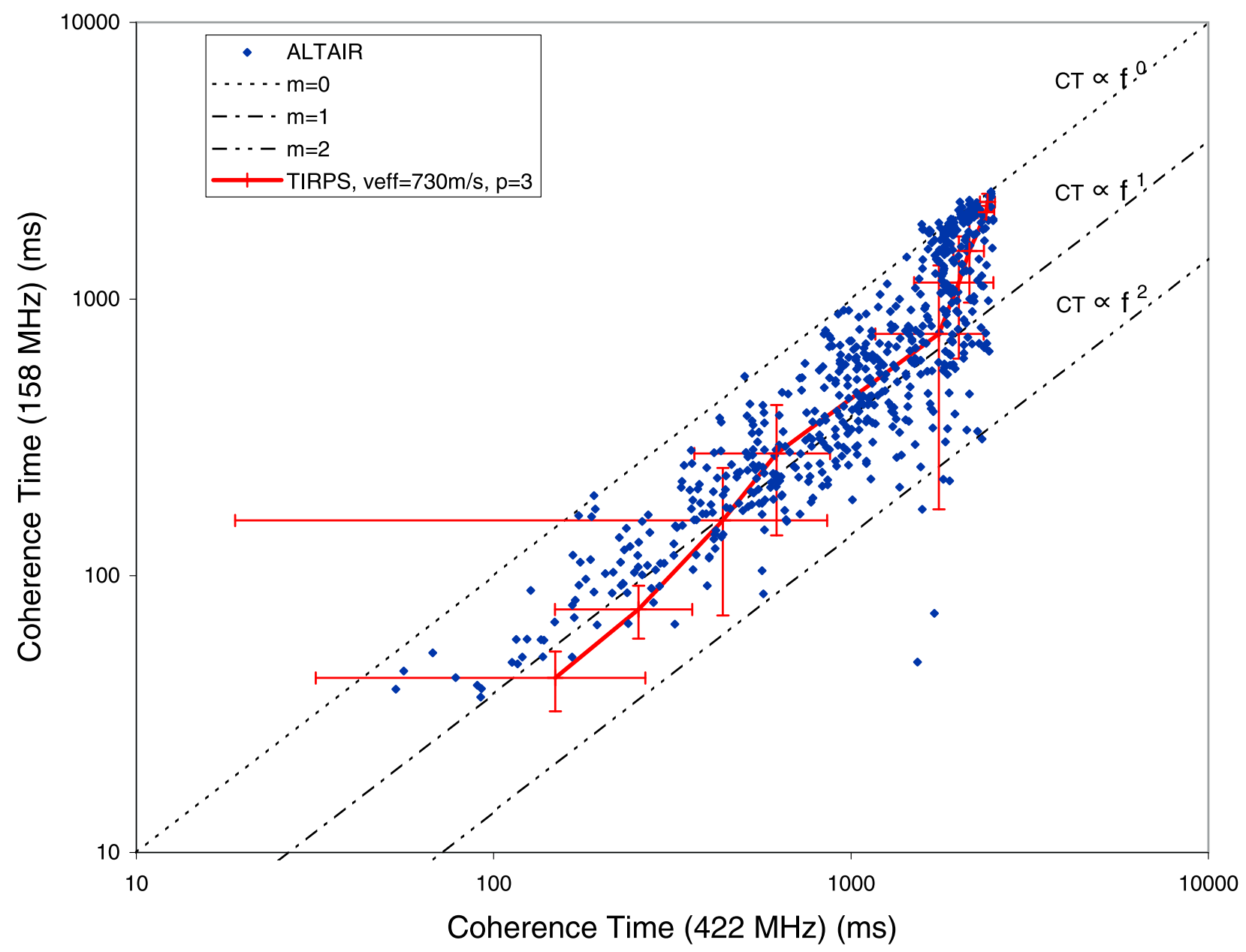

Figure 7. Coherence times of CSFs (points) measured by ALTAIR at $158 \mathrm{MHz}$ plotted against those simultaneously measured at $422 \mathrm{MHz}$. The solid red line represents TIRPS simulation (mean \pm standard deviation of 10 runs) using $v_{\text {eff }}=730 \mathrm{~m} \mathrm{~s}^{-1}, p=3$, and $L_{o}=10 \mathrm{~km}$.

$v_{\text {eff }}=730 \mathrm{~m} \mathrm{~s}^{-1}$. Once again the TIRPS model faithfully reproduces the experimental results.

\section{Summary and Conclusions}

[23] The Wideband Ionospheric Distortion Experiment (WIDE) using the ALTAIR radar sought to measure the equatorial transionospheric propagation channel under a wide variety of ionospheric conditions and to relate those measurements to the design of wideband radio systems, especially space-based wideband synthetic aperture radars. However, from an early stage it was recognized that a dual approach of measurement and modeling was required to best achieve these aims. This led to the implementation of the Trans-Ionospheric Radio Propagation Simulator (TIRPS). By making measurements and refining this model we have been able to better understand the impact that the ionosphere has on the operation of such sophisticated systems. So far all of our data collects have occurred during the years of sunspot minimum and our experiment lacks the bandwidth to fully elucidate the issue of coherency bandwidth. As we move toward sunspot maximum, the coherency bandwidths and times are likely to reduce and this should mitigate this limitation.

[24] In the work of Cannon et al. [2006] we described the WIDE experiment, our first results and provided examples of channel scattering functions and estimates of the coherency bandwidths and times. In the work of Rogers et al. [2009], a companion to this paper, we describe the TIRPS model in some detail and undertake some detailed cross comparisons between the measure- 


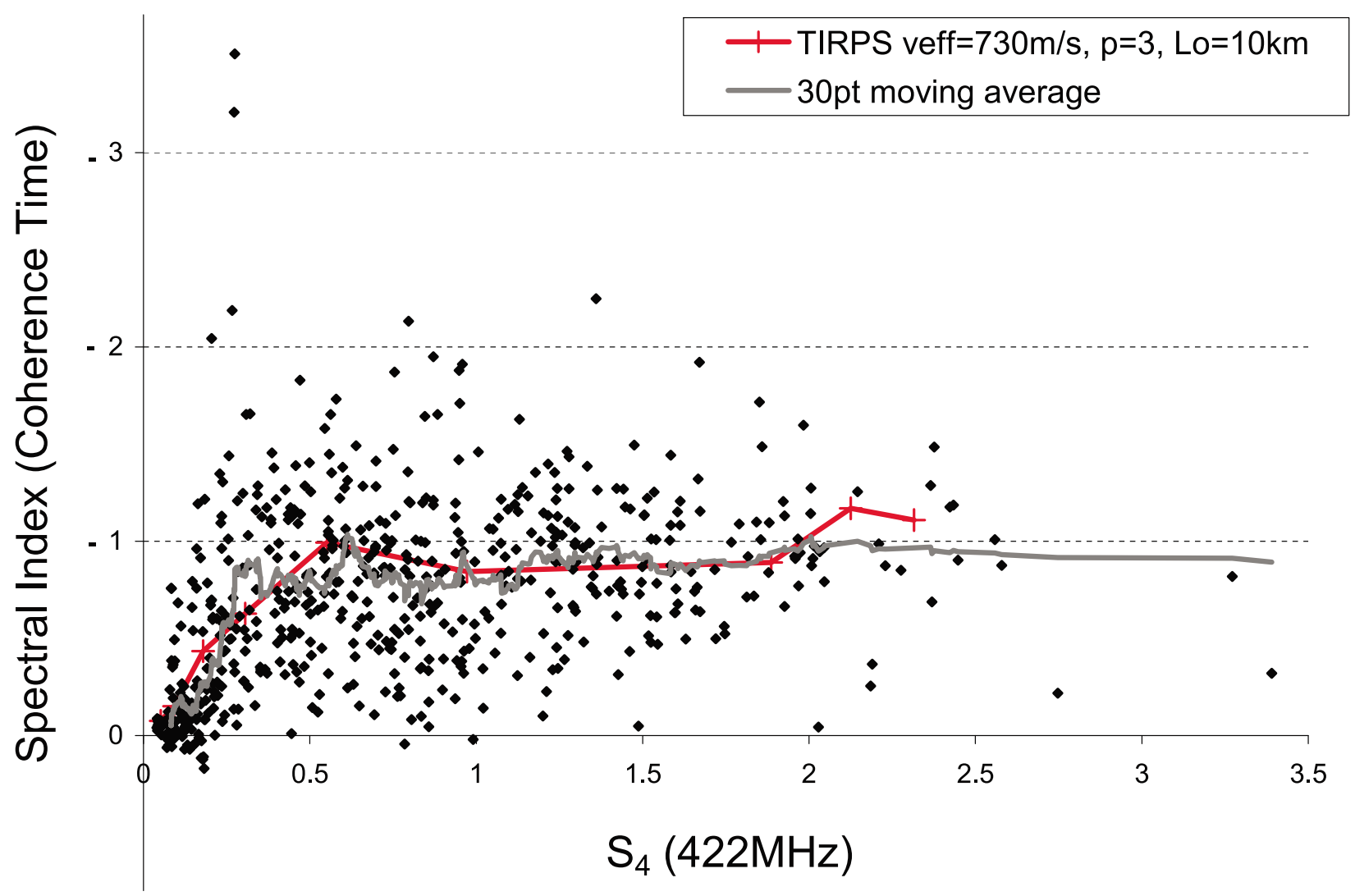

Figure 8. Spectral index of Doppler spread as a function of the $422 \mathrm{MHz} \mathrm{S}_{4}$ index. The gray line represents a 30-point moving average. The red line indicates TIRPS simulation averages (of 10 runs) using $v_{\text {eff }}=730 \mathrm{~m} \mathrm{~s}^{-1}, p=3$, and $L_{o}=10 \mathrm{~km}$.

ments and the model, thereby providing credence to that model.

[25] In this paper we have extended that cross comparison to look in more detail at the issue of coherency bandwidth and times, for a wide range of conditions from weak scintillation to strong scintillation, under conditions of strong focusing. We have demonstrated a high degree of correlation between the model and the measurements and have demonstrated that the model is able to characterize the CT of the equatorial ionosphere on wideband systems, operating in the frequency range 150 to $450 \mathrm{MHz}$. While our CB intercomparison is broadly in agreement, the lack of measurement bandwidth means that we are unable to fully validate this aspect of our model.

[26] We have also recognized the need to have a practical strategy for running such a model and we have demonstrated that rather than using the integrated strength of turbulence parameter $C_{k} L$, we are able to use the common $\mathrm{S}_{4}$ parameter and produce meaningful results. We note however, that we have demonstrated good correlation between our model and measurements when using the $422 \mathrm{MHz} \mathrm{S}_{4}$ derived from our two-way measurements. It remains to be seen if this can be extended to using $\mathrm{S}_{4}$ from other sensors, particularly from GPS receivers.

[27] Our results may be summarized as follows:

[28] 1. The measured CT decreased exponentially with $\mathrm{S}_{4}(422 \mathrm{MHz})$ up to the Rayleigh limit of $\mathrm{S}_{4}=2.2$. The CT decreased below $100 \mathrm{~ms}$ at the highest $\mathrm{S}_{4}(>1.5)$ and highest $v_{\text {eff }}$.

[29] 2. The TIRPS simulations best matched this trend using a phase screen with $p=3$. A higher spectral gradient $(p=4)$ was required to simulate the CT for $\mathrm{S}_{4}>2.2$, (strong focusing conditions).

[30] 3. There was a decrease in CT for higher values of effective scan velocity $\left(v_{\text {eff }}\right)$ and the simulations provided a good fit to the CT trend with $\mathrm{S}_{4}$ when an appropriate value of $v_{\text {eff }}$ was used in the simulation.

[31] 4. The coherence bandwidth (CB) was generally limited by the radar chirp bandwidth except for a small number of $158 \mathrm{MHz}$ CSFs above an $\mathrm{S}_{4(422 \mathrm{MHz})}$ of 1.3, where the $\mathrm{CB}$ reduced to as little as $0.7 \mathrm{MHz}$. This 
behavior is again broadly reproduced in the TIRPS simulations (but see comments above).

[32] 5. To extend these results to other radio frequencies we presented the frequency dependences of CT and $\mathrm{S}_{4}$ and their variation with $\mathrm{S}_{4}$ at the higher frequency.

[33] 6. The spectral indices of the CT measurements vary widely about a mean of about -0.9 , and this is reproduced by the TIRPS model.

[34] 7. The spectral index of the $S_{4}$ measurements decreases linearly with increasing $\left.\mathrm{S}_{4(422} \mathrm{MHz}\right)$, ranging between around 1.5 at low $\mathrm{S}_{4}$ to -0.3 at the highest $\mathrm{S}_{4}$. The TIRPS simulations closely follow this trend, for $p=3$ and with an appropriate $v_{\text {eff }}$ and elevation.

[35] Acknowledgments. The UK contribution to this program has been conducted with funding from the United Kingdom Ministry of Defence Science and Technology program. The MIT Lincoln Laboratory contribution was sponsored by the U.S. Department of the Army under Air Force contract FA8721-05-C-0002. Opinions, interpretations, conclusions, and recommendations are those of the authors and are not necessarily endorsed by the United States government. The authors would like to thank Paul Kossey for his unfailing support in the early stages of this UK-U.S. collaborative program.

\section{References}

Aarons, J. (1982), Global morphology of ionospheric scintillation, Proc. IEEE, 70(4), 360-377.

Aarons, J. (1993), The longitudinal morphology of equatorial F-layer irregularities relevant to their occurrence, Space Sci. Rev., 63(3-4), 209-243.

Belcher, D. P., and N. C. Rogers (2009), Theory and simulation of ionospheric effects on synthetic aperture radar, IET Radar Sonar Navig., 3(5), 541-551, doi:10.1049/iet-rsn.2008.0205.

Bramley, E. N. (1977), The accuracy of computing ionospheric radio-wave scintillation by the thin phase-screen approximation, J. Atmos. Terr. Phys., 39, 367-373.

Cannon, P. S., and P. Bradley (2003), Ionospheric propagation, in Propagation of Radio Waves, 2nd ed., edited by L. W. Barclay, chap. 16, pp. 313-333, Inst. of Electr. Eng., London, U. K.

Cannon, P. S., K. Groves, D. J. Fraser, W. J. Donnelly, and K. Perrier (2006), Signal distortion on VHF/UHF transionospheric paths: First results from the Wideband Ionospheric Distortion Experiment, Radio Sci., 41, RS5S40, doi:10.1029/2005RS003369.

Coster, A. J., E. M. Gaposchkin, L. E. Thornton, G. R. Krumholz, and T. A. Cott (1993), Ionospheric effects in satellite tracking, in Proceedings of 7th Ionospheric Effects Symposium, edited by J. M. Goodman, pp. 80-87, Natl. Tech. Inf. Serv., Springfield, Va.

Coster, A. J., L. E. Thornton, T. DeVere, J. Randolph, and B. Wilson (2002), Analysis of ionospheric errors at space surveillance radars, in Proceedings of 10th International Ionospheric Effects Symposium, edited by J. M. Goodman, pp. 475-483, Natl. Tech. Inf. Serv., Springfield, Va.

Davies, K. (1990), Ionospheric Radio, IEE Electromagn. Waves Ser., vol. 31, Peter Perigrinus, London, U. K.

Fremouw, E. J., and A. Ishimaru (1992), Intensity scintillation index and mean apparent radar cross section on monostatic and bistatic paths, Radio Sci., 27(4), 539-543.

Knepp, D. L. (1983a), Multiple phase-screen calculation of the temporal behavior of stochastic waves, Proc. IEEE, 71(6), $722-737$.

Knepp, D. L. (1983b), Analytical solution for the two-frequency mutual coherence function for spherical wave propagation, Radio Sci., 18(4), 535-549.

Levy, M. (2000), Parabolic Equation Methods for Electromagnetic Wave Propagation, IEE Electromagn. Waves Ser., vol. 45, Inst. of Electr. Eng., London, U. K.

Nakagami, M. (1960), The m-distribution, a general formula of intensity distribution of rapid fading, in Statistical Methods in Radio Wave Propagation: Proceedings of a Symposium Held at the University of California, edited by W. Hoffman, pp. 3-36, Pergamon, New York.

Rino, C. L. (1979), A power law phase screen model for ionospheric scintillation: 1. Weak scatter, Radio Sci., 14(6), $1135-1145$.

Rino, C. L. (1980), Numerical computations for a onedimensional power law phase screen, Radio Sci., 15(1), 41-47.

Rogers, N. C., P. S. Cannon, and K. M. Groves (2009), Measurements and simulation of ionospheric scattering on VHF and UHF radar signals: Channel scattering function, Radio Sci., 44, RS0A07, doi:10.1029/2008RS004033.

van de Kamp, M., P. S. Cannon, and M. Terkildsen (2009), Effect of the ionosphere on defocusing of space-based radars, Radio Sci., 44, RS1003, doi:10.1029/2007RS003808.

P. S. Cannon and N. C. Rogers, Centre for RF Operational Environments, QinetiQ, Malvern WR14 3PS, UK. (ncrogers@ qinetiq.com)

K. M. Groves, Air Force Research Laboratory, Hanscom Air Force Base, MA 01731, USA. 